\title{
IN SILICO INVESTIGATION OF ECHINODERMATA SECONDARY METABOLITES AS HUMAN IMMUNODEFICIENCY VIRUS TYPE 1 (HIV-1) REVERSE TRANSCRIPTASE INHIBITORS
}

\author{
NURRAHMA NAZWIR, ARRY YANUAR, REZI RIADHI SYAHDI* \\ Department of Pharmaceutical Chemistry, Biomedical Computation Laboratory, Faculty of Pharmacy, Universitas Indonesia, Depok \\ 16424, Indonesia. Email: rezi.riadhi@farmasi.ui.ac.id
}

Received: 02 October 2019, Revised and Accepted: 24 December 2019

\section{ABSTRACT}

Objective: Human immunodeficiency virus (HIV) targets the immune system and weakens immune surveillance and defenses against infections, leading to acute immunodeficiency syndrome. Recent trends in drug discovery from natural sources emphasize investigations of compounds from marine ecosystems.

Methods: In this study, we compiled a database of chemical compounds from echinoderms and virtually screened for those that inhibit HIV-1 reverse transcriptase (RT). The database was generated from literature searches. Virtual screening analyses for inhibitors of HIV-1 RT were then performed using AutoDock software.

Results: Based on screening results, the top thirteen ranked compounds were nobilisidenol B, Ech_005, 17-deoxyholothurinogenin, 22,25-oxidoholothurinogenin, Ech_022, Ech_026, Ech_021, nobilisidenol A, Ech_025, 5 $\alpha$-cholest-8(14)-ene-3ß,7 $\alpha$-diol, astropecten A, Ech_004, and phrygiasterol.

Conclusion: The present in silico screening analyses of compounds from marine ecosystems can be used to identify candidate compounds with high potential as drugs for the treatment of refractory HIV infections.

Keywords: Echinoderms, Human immunodeficiency virus, Reverse transcriptase inhibitor.

(c) 2020 The Authors. Published by Innovare Academic Sciences Pvt Ltd. This is an open access article under the CC BY license (http://creativecommons. org/licenses/by/4. 0/) DOI: http://dx.doi.org/10.22159/ijap.2020.v12s1.FF006

\section{INTRODUCTION}

Marine environments are known sources of unique and potent compounds with pharmacological properties such as antitumor, anti-inflammatory, analgesic, immunomodulatory, anti-allergy, and antiviral activities [1]. Yet compounds from marine environments have not yet been investigated extensively and have only been researched, collected, extracted, and explored chemically since the mid-late 1960s. In comparison, compounds from terrestrial environments have been exploited by humans for more than 3000 years [2]. Hence, marine resources may provide alternative candidate compounds for new active drugs for the treatment of various diseases, such as human immunodeficiency virus type 1 (HIV-1), a disease that remains a major global public health issue [3]. Among marine organisms, echinoderms are exclusively found in salt water, with no fresh water or terrestrial members or similar organisms have been identified [4]. Hence, novel compounds/ new chemical entities that are peculiar to marine environments are likely to differ from those of terrestrial organisms. Previous researches have shown echinoderm compounds with reported antiviral activities include imbricantine from Dermasterias imbricata [5,6], four new sulfated polar steroids from the Far Eastern starfish Leptasterias ochotensis [7], hedathiosulfonic acids A and B from Echinocardium cordatum [6,8], lysastroside from Lysastrosoma anthosticta [6,9], and ten saponin compounds from Certonardoa semiregularis [10]. In silico analyses may offer valuable research information and reduce the costs of drug development by reducing sample numbers in in vitro and in vivo studies [11]. Several studies have shown that virtual screening could be performed for marine compounds to target various macromolecules such as epidermal growth factor receptor-TK, vascular EGFR-2, mouse double minute 2 , and procaspase-3 [12-14]. Herein, we performed in silico molecular docking experiments to identify the most promising compounds that can be isolated from echinoderms as HIV-1 reverse transcriptase (RT) inhibitors.

\section{METHODS}

\section{Ligand preparation}

Two dimensional (2D) structures of echinoderms bioactive compounds were compiled by data mining numerous journals. Subsequently, three dimensional (3D) structures were created in MarvinSketch [15] using mmff94 force field and strict level optimization limits. The prepared ligands were saved in *.pdb files and were converted to *.pdbqt files.

\section{Macromolecule preparation}

Macromolecules were downloaded from the Protein Data Bank using 3LP1 code [16]. Macromolecules were then prepared by separating from solvents, ligands, and other nonstandard residues using AutoDockTools [17]. We then optimized these by deleting water molecules, adding nonpolar hydrogen atoms, charges, and force fields, and by minimizing the structures using AutoDockTools. Prepared macromolecules were saved in *.pdb files and were then converted to *.pdbqt files.

\section{Docking parameter optimization}

In silico docking was performed using AutoDock 4.0 [17], which was run automatically through PyRx [18], and the results were visualized using PyMOL [19] and LigandScout [20]. Molecular docking parameters were optimized by redocking nevirapine. Positive controls included nevirapine, delavirdine, efavirenz, rilpivirine, and etravirine, which are recommended by the United States Food and Drug Administration (FDA) for the treatment of HIV-1 [21].

\section{Virtual screening}

Virtual screening was performed with HIV-1 RT using the AutoDock function of PyRx. The parameters were as follows: Search space surrounding the binding pocket, $18.75 \times 18.75 \times 18.75 \AA$; maximum number of generations, 27,000; maximum number of energy of 


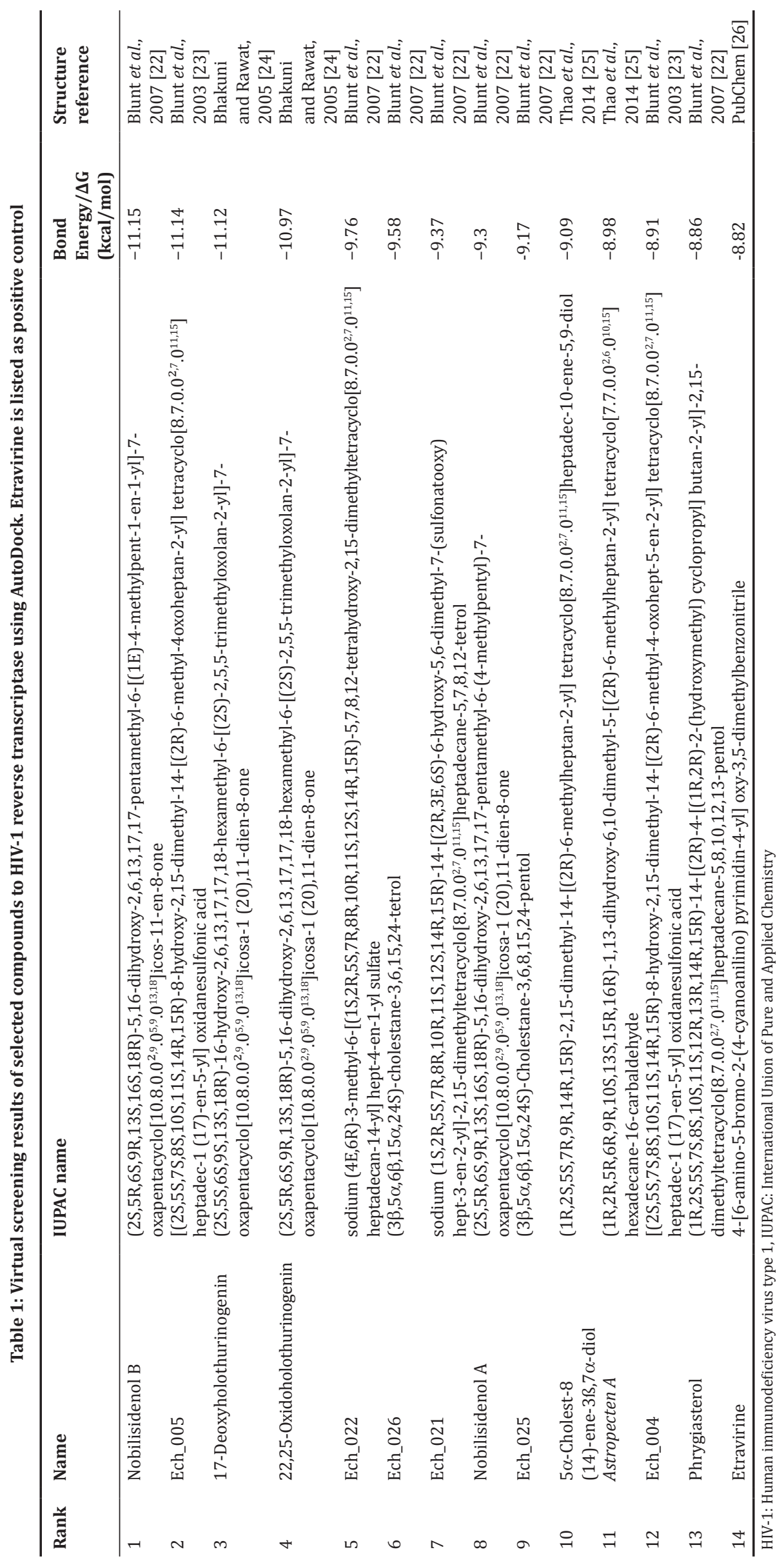


evaluations, 250,000; number of individuals in population, 150; number of genetic algorithm runs, 10; rate of gene mutation, 0.02; and rate of crossover, 0.8 . These parameters were optimized in previous studies $[27,28]$.

\section{RESULTS AND DISCUSSION}

\section{Macromolecule preparation}

We initially prepared macromolecules by searching the protein database and deleting nonstandard residues. The nonstandard residues nevirapine ligand, manganese ion (II), and LP8 were then separated from structures as identifier molecules. Hydrogen atoms that are not generally identified in crystal structures were then added to manipulate hydrogen bonding with added ligands. AutoDock force field was then applied with Gasteiger charges as a common default for docking in AutoDock applications. We then performed energy minimization to identify geometrical conformations with the least optimal energy so that the structures could be considered stable as docking targets. The coordinates of the nevirapine binding site were determined as a positive control and the binding site was defined as $\mathrm{x}=10.350, y=14.076$, and $\mathrm{z}=18.252$. The grid was set at $50 \times 50 \times 50$ units at $0.375 \AA$. This coordinate determination for the receptor target corresponded with previous research [28].

\section{Ligand database preparation}

The ligand database that was developed in this experiment comprised ligand names from the International Union of Pure and Applied Chemistry (IUPAC), 2D and 3D structures, species names from which the compounds were extracted/isolated, and pharmacological actions (if any) and references. Data from various journals were obtained from the PubChem Open Chemistry Database [26], the ChemSpider Search and Share Chemistry [29] and other resources in *.sdf and *.mol formats. Hydrogen atoms were added because the software scoring function requires polar and nonpolar hydrogen atoms. Gasteiger charges and AutoDock force fields were added because they are generally used in AutoDock software. Some of the compounds that were compiled from journals already have names, whereas others have only IUPAC names. To facilitate docking and analysis, ligand nomenclature for unnamed compounds was standardized as Ech_XXX, where XXX are three-digit numbers of compounds that were sorted based on literature reviews.

\section{Analysis of virtual screening results}

Virtual screening was performed for 127 compounds that were identified by data mining of several journals. Virtual screening tests of RT inhibitory activities of ligands were sorted and ranked based on $\Delta \mathrm{G}$ values. Compounds with the lowest $\Delta \mathrm{G}$ values were placed in the first rank. The results are listed in Table 1.

Based on our screening results, the top thirteen ranked compounds were nobilisidenol B, Ech_005, 17-deoxyholothurinogenin, 22,25-oxidoholothurinogenin, Ech_022, Ech_026, Ech_021, nobilisidenol A, Ech_025, $5 \alpha$-cholest-8(14)-ene-3ß,7 $\alpha$-diol, astropecten A, Ech_004, and phrygiasterol. These compounds were selected for further analysis based on energy bond values that were superior to those of the positive control (Table 3). Etravirine was identified as a non-nucleoside RT inhibitor (NNRTI) and had bond energy of -8.82 $\mathrm{kcal} / \mathrm{mol}$ [28]. All compounds were selected based on their anti-HIV activities. Based on the literature searches and reviews, these thirteen compounds have not been identified as anti-HIV or RT inhibitors previously.

\section{Visualization of virtual screening results}

After identifying top compounds based on ranking, their active sites were observed from validation and virtual screening of docking results and were visualized using LigandScout. After analyzing and visualizing chemical bonds and interactions between cocrystals or positive controls and receptor targets, interactions between validated bioactive compounds from echinoderms and RT were analyzed and visualized in terms of hydrogen bond lengths using PyMOL. In these experiments, the most important interactions were weak intermolecular forces comprising hydrogen bonds and hydrophobic interactions. These are key properties for stabilizing ligands in open conformations of protein structures. Specifically, hydrogen bonds are formed through interactions between hydrogen atoms that are covalently bound to electronegative donor atoms with electronegative acceptor atoms. These interactions are important for proteins and occur predominantly between $\mathrm{NH}$ and $\mathrm{CO}$ groups of main $\alpha$-helical chains. Hydrogen bonds between macromolecules and their ligands (proteins, nucleic acids, substrates, effectors, and inhibitors) contribute directionality and specificity of interactions and serve as fundamental mediators of molecular recognition [30-33].

In 2009, Eugene E. Kwan classified hydrogen bonds with donor-acceptor distances of $2.2-2.5 \AA$ as "strong, mostly covalent," of $2.5-3.2 \AA$ as "medium, mostly electrostatic," and of 3.2-4.0 $\mathrm{\text {as }}$ "weak, electrostatic" [33]. This hydrogen bonds classification is defined in Table 2.

Analysis and visualization of interactions between compounds from echinoderms and macromolecule targets using AutoDock 2D and 3D structures of docking results were modeled using LigandScout. As shown in Fig. 1, the highest-ranked compound nobilisidenol B was docked to 3LP1. This triterpene was isolated from the sea cucumber Holothuria nobilis [22]

The LigandScout analysis and visualization of 2D and 3D structures identified two hydrogen bonds. In one of these, nobilisidenol B provides a hydrogen bond donor and an acceptor from the same amino-acid residue Tyr318. The nobilisidenol B functional group that acted as both H-bond donor (HB1) and acceptor (HB2) comprises hydroxyl groups of the phenanthrene core, with distances of $3.2 \AA$ and $2.8 \AA$, respectively. These distances were measured using PyMOL and showed that HB1 is a medium H-bond and HB2 is a weak H-bond. Moreover, hydrophobic interactions were identified between nobilisidenol B and the aminoacid residues Ile180, Leu100, Tyr181, Val106, and Val179.

Interactions between the positive control and the thirteen compounds from echinoderms and amino-acid residues are shown in Table 3.

Some amino-acid residues that play important roles as NNRTI binding sites are listed in Table 3. Among these, Lys101 is considered important because it has the most interactions of all 3LP1 amino-acid residues, which participates in hydrogen and ionic bonds with some compounds from echinoderms more than with other amino-acid residues. In a book published in 2006, Skowron and Ogden wrote about a number

Table 2: Classification of hydrogen bond strength

\begin{tabular}{llll}
\hline Indicator & Strong & Medium & Weak \\
\hline Bond energy $(\mathrm{kcal} / \mathrm{mol})$ & $14-40$ & $4-14$ & $0-4$ \\
Interaction type & $\begin{array}{l}\text { Mostly } \\
\text { covalent }\end{array}$ & $\begin{array}{l}\text { Mostly } \\
\text { electrostatic }\end{array}$ & Electrostatic \\
A---B $(\AA)$ & $2.2-2.5$ & $2.5-3.2$ & $3.2-4.0$ \\
H...B $(\AA)$ & $1.2-1.5$ & $1.5-2.2$ & $2.2-3.2$ \\
Bond angle $\left({ }^{\circ}\right)$ & $175-180$ & $130-180$ & $90-150$ \\
\hline
\end{tabular}

Source: Kwan, E. E., 2009 [33]

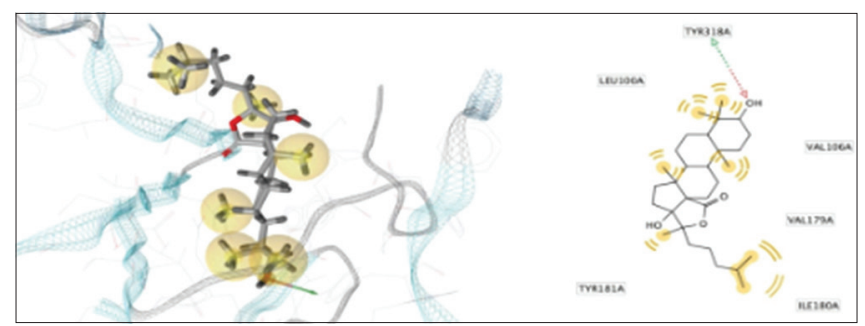

Fig. 1: Visualization of interactions between nobilisidenol $B$ and 3LP1

Source: LigandScout 


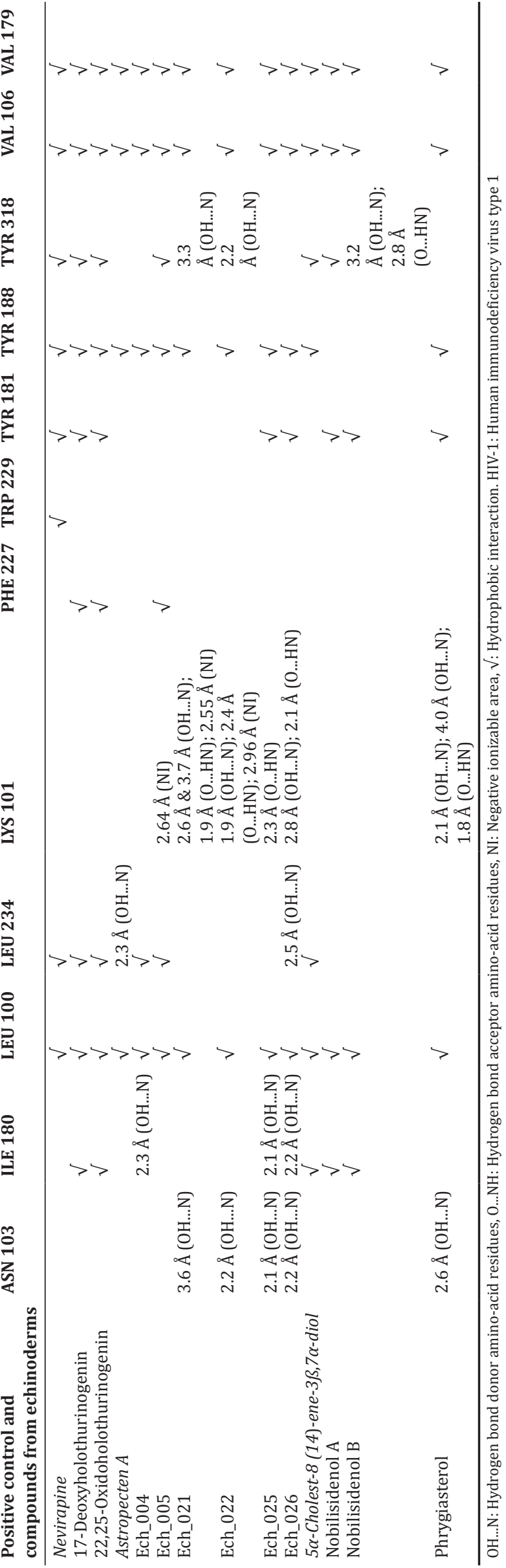

of hydrogen-bonding interactions between NNRTIs and protein mainchain atoms. They suggest that the most commonly observed hydrogen bonds involve carbonyl groups of Lys101 [34]. Hydrogen bonding with Lys103 and Pro236 main-chain atoms has also been observed. In addition, we observed hydrogen-bonding interactions between Lys101 and the selected compounds Ech_021, Ech_022, Ech_025, Ech_026, and phrygiasterol. Ionic interactions were also observed between the $\mathrm{NH}_{3+}$ group of Lys101 and S042- groups of the compounds Ech_005, Ech_021, and Ech_022.

Interactions between Lys101 and $\mathrm{SO}_{4}{ }^{2-}$ ions deserve special attention because they have not been recognized in the previous studies of NNRTI binding sites. Accordingly, no NNRTI drugs that were approved by the FDA have reported ionic-bonding interactions with Lys101. Therefore, due to $\mathrm{SO}_{4}{ }^{2-}$ groups that participate in ionic interactions with Lys101, the echinoderm compounds Ech_005, Ech_021, and Ech_022 are promising candidate NNRTI drugs, along with the other top thirteen ranked compounds.

\section{CONCLUSION}

Database and literature searches of chemical compounds from echinoderms revealed 127 candidate compounds. Subsequent virtual screening for RT inhibitory activities using AutoDock identified nobilisidenol B, Ech_005, 17-deoxyholothurinogenin, 22,25-Oxidoholothurinogenin, Ech_022, Ech_026, Ech_021, nobilisidenol A, Ech_025, $5 \alpha$-cholest-8(14)-ene-3ß,7 $\alpha$-diol, astropecten A, Ech_004, and phrygiasterol as high potential candidate anti-HIV drugs.

\section{CONFLICTS OF INTEREST STATEMENT}

The authors do not have any conflicts of interest to declare.

\section{REFERENCES}

1. Newman DJ, Cragg GM. Marine natural products and related compounds in clinical and advanced preclinical trials. J Nat Prod 2004;67:1216-38.

2. Fenical W. Marine pharmaceuticals: Past, present, and future. Oceanography 2006;19:110-9.

3. World Health Organization. HIV/AIDS Fact Sheet; 2016. Available from: http://www.who.int/mediacentre/factsheets/fs360/en.

4. Prothero DR. Bringing Fossils to Life: An Introduction to Paleobiology. USA: Columbia University Press; 2013.

5. Jha RK, Zi-Rong X. Biomedical compounds from marine organisms. Mar Drugs 2004;2:123-46.

6. Carte BK. Biomedical potential of marine natural products. Bioscience 1996;46:271-86.

7. Malyarenko TV, Malyarenko Vishchuk OS, Ivanchina NV, Kalinovsky AI, Popov RS, Kicha AA. Four new sulfated polar steroids from the far eastern starfish Leptasterias ochotensis: Structures and activities. Mar Drugs 2015;13:4418-35.

8. Kita M, Watanabe M, Takada N, Suenaga K, Yamada K, Uemura D. Hedathiosulfonic acids A and B, novel thiosulfonic acids from the deepsea urchin Echinocardium cordatum. Tetrahedron 2002;58:6405-12.

9. Levina EV, Andriyashchenko PV, Kalinovsky AI, Dmitrenok PS, Stonik VA, Prokof NG. Steroid compounds from the starfish Lysastrosoma anthosticta collected in the sea of Japan. Russ Chem Bull 2002;51:535-9.

10. Wang W, Li F, Alam N, Liu Y, Hong J, Lee CK, et al. New saponins from the starfish Certonardoa semiregularis. J Nat Prod 2002;65:1649-56.

11. Maithri G, Manasa B, Vani SS, Narendra A, Harshita T. Computational drug design and molecular dynamic studies a review. Int J Biomed Data Min 2016;6:1-7.

12. Yanuar A, Chavarina KK, Syahdi RR. Molecular dynamic simulation analysis on marine fungi compounds against EGFR and VEGFR-2 inhibitory activity in non-small cell lung cancer. J Young Pharm 2018;10:S25.

13. Yanuar A, Pratiwi I, Syahdi RR. In silico activity analysis of saponins and 2, 5-piperazinedione from marine organism against murine double minute-2 inhibitor and procaspase-3 activator. J Young Pharm 2018;20:S16.

14. Watty M, Syahdi RR, Yanuar A. Database compilation and virtual 
screening of secondary metabolites derived from marine fungi as epidermal growth factor receptor tyrosine abstract kinase inhibitors. Asian J Pharm Clin Res 2017;10:142-7.

15. MarvinSketch (version 6.2.2), Calculation Module Developed by Chem Axon 2014. Available from: http:/www.chemaxon.com/products/ marvin/marvinsketch.

16. Su HP, Yan Y, Prasad GS, Smith RF, Daniels CL, Abeywickrema PD, et al. Structural basis for the inhibition of RNase H activity of HIV-1 reverse transcriptase by $\mathrm{RNase} \mathrm{H}$ active site-directed inhibitors. J Virol 2010;84:7625-33

17. Morris GM, Huey R, Lindstrom W, Sanner MF, Belew RK, Goodsell DS, et al. Auto dock4 and auto dock tools4: Automated docking with selective receptor flexibility. J Comput Chem 2009;30:2785.

18. Wolf LK. PyRx. Chem Eng New 2009;87:31

19. DeLano WL. The PyMOL Molecular Graphics System, Version 0.99. New York: Schrödinger, LLC; 2002.

20. Wolber G, Langer T. Ligand scout: 3-D pharmacophores derived from protein-bound ligands and their use as virtual screening filters. J Chem Inf Model 2005;45:160-9.

21. De Clercq E. Anti-HIV drugs: 25 compounds approved within 25 years after the discovery of HIV. Int J Antimicrob Agents 2009;33:307-20.

22. Blunt JW, Copp BR, Hu WP, Munro MH, Northcote PT, Prinsep MR. Marine natural products. Nat Prod Rep 2007;24:31-86.

23. Blunt JW, Copp BR, Munro MH, Northcote PT, Prinsep MR. Marine natural products. Nat Prod Rep 2003;20:1-48

24. Bhakuni DS, Rawat DS. Bioactive Marine Natural Products. India: Springer and Anamaya Publisher; 2005.

25. Thao NP, No JH, Luyen BT, Yang G, Byun SY, Goo J, et al. Secondary metabolites from Vietnamese marine invertebrates with activity against Trypanosoma brucei and T. cruzi. Molecules 2014;19:7869-80.

26. Im S, Thiessen PA, Bolton EE, Chen J, Fu G, Gindulyte A, et al. PubChem substance and compound databases. Nucleic Acids Res 2016;44: D1202-13.

27. Yanuar A, Mun'im A, Lagho AB, Syahdi RR, Rahmat M, Suhartanto H. Medicinal plants database and three dimensional structure of the chemical compounds from medicinal plants in Indonesia. Int J Comput Sci Issues 2011;8:180-3.

28. Syahdi RR, Mun'im A, Suhartanto H, Yanuar A. Virtual screening of Indonesian herbal database as HIV-1 reverse transcriptase inhibitor. Bioinformation 2012;8:1206-10.

29. Pence HE, Williams A. Chemspider: An online chemical information resource. J Chem Educ 2010;87:1123-4.

30. Hubbard RE, Haider MK. Hydrogen Bonds in Proteins: Role and Strength. UK: ELS; 2010.

31. Kusuma SA, Manan WS, Budiman F. Inhibitory effect of red piper betel leaf ethanol extract (Piper crocatum Ruiz and Pav.) against Trichomonas vaginalis trophozoites in vitro. Asian J Pharm Clin Res 2017;10:311-4

32. Adiga R. Molecular docking of Hyrtimomine A-K from marine sponge Hyrtios spp. as anticancer target of phospoinositide-dependent kinase 1 . Asian J Pharm Clin Res 2019;12:130-5.

33. Kwan EE. An Introduction to Hydrogen Bonding. Presented at an Evans Group Afternoon Seminar; 2009.

34. Skowron G, Ogden R. Transcriptase Inhibitors in HIV/AIDS Therapy. New Jersey: Humana Press Inc.; 2006. 